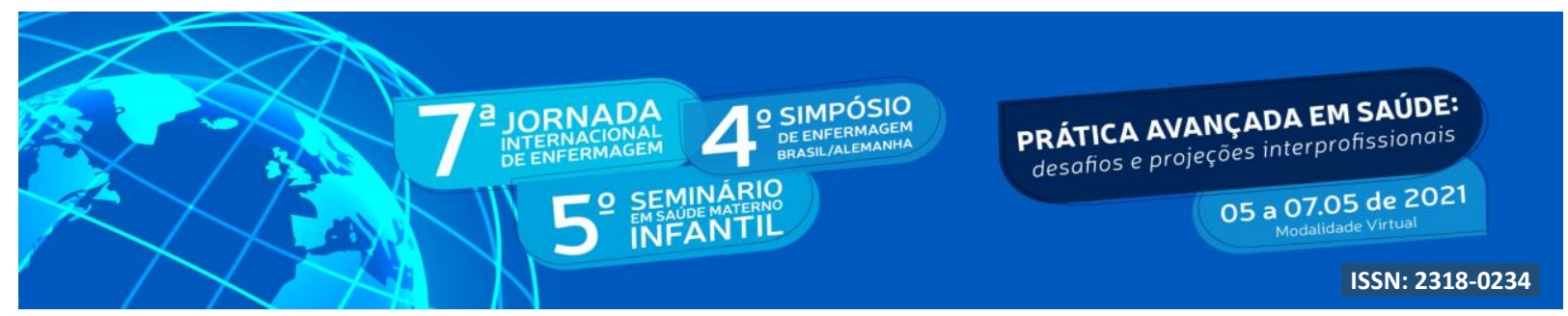

DOI: http://doi.org/10.48195/jie2021-002

\title{
SAÚDE DAS MULHERES EM CABO VERDE
}

\author{
Carolina Henriques'; João Frade²; Paulo Santos ${ }^{3}$
}

\begin{abstract}
RESUMO
Falarmos da saúde das mulheres é falarmos da saúde genésica das populações, já que as mulheres são as principais responsáveis pelo cuidado ao outro. Neste estudo procurámos conhecer a saúde e o bemestar das mulheres em cabo verde, seja a nível físico, seja do ponto de vista emocional, através de um estudo quantitativo e descritivo, dirigido a 104 mulheres cabo-verdianas. Os dados mostram-nos que é necessário investirmos na saúde da mulher em Cabo-Verde, já que as experiências de doença nas mulheres tendem a ser ocultadas e vivenciadas em silêncio, parecendo que a mulher carrega o fardo da resignação e sofrimento, já que as mulheres cabo-verdianas tendem a continuar como de costume, guardando esse facto somente para elas.
\end{abstract}

Palavras-chave: Saúde; Mulheres; Cabo-Verde.

\begin{abstract}
Talking of women's health is to reflect of the population's health, since women are primarily responsible for caring for others. In this study, we sought to understand the health and well-being of women in Cape Verde, namely physically and emotionally, through a quantitative and descriptive study, addressed to 104 Cape Verdean women. The data shows us that it is necessary to invest in women's health in Cape-Verde, since the experiences of illness in women tend to be hidden and livedin silence, it seems that women bear the burden of resignation and suffering, since Cape Verdean women tend to continue with their daily life as usual, keeping this fact to themselves.
\end{abstract}

Key Words: Health; Women; Cape Verde.

\footnotetext{
${ }^{1}$ Carolina Miguel da Graça Henriques, Center for Innovative Care and Health Technology (ciTechCare), Health Sciences School, Polytechnic Institute of Leiria, Leiria, Portugal. carolina.henriques@ipleiria.pt; orcid.org/00000002-0904-8057

2 João Manuel da Graça Frade, Center for Innovative Care and Health Technology (ciTechCare), Health Sciences School, Polytechnic Institute of Leiria, Leiria, Portugal. joão.frade@ipleiria.pt; orcid.org/0000-00024947-1052

${ }^{3}$ Paulo Alexandre Figueiredo dos Santos, Center for Interdisciplinary Research in Health (CIIS), Universidade Católica Portuguesa, Lisboa, Portugal. orcid.org/0000-0Q01-7409-1011
} 


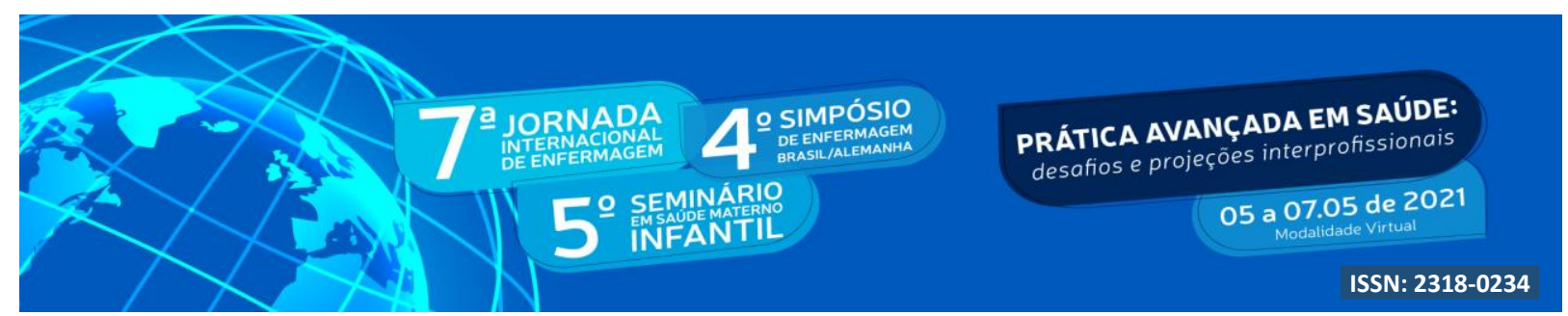

\section{INTRODUÇÃ̃O}

Até há pouco tempo, a única perspetiva e quase exclusiva leitura existente da saúde das mulheres, das suas doenças ou do seu estado de bem-estar, baseava-se essencialmente nas questões reprodutivas e nas funções que lhes estão associadas.

Esta forma de ver a saúde das mulheres não fez mais do que traduzir no domínio da saúde a leitura dominante dos papéis sociais das mulheres, cuja tarefa mais importante é a de reprodução, esquecendo as outras que lhe estão ligadas: que os seres que nascem não seriam humanos se não houvesse, para além do trabalho de reprodução, um trabalho de criação do humano que não é simplesmente material (calor, abrigo, comida, higiene), mas também simbólico (afeto, vinculação, contacto).

Hoje a OMS (2019) assume que é necessário valorizar os cuidados a todas as mulheres, procurando articular as diferentes perspetivas que a sua saúde encerra: o cuidado de si, os cuidados aos outros e o cuidar do espaço publico enquanto espaço de vida.

As mulheres representam 51,2\% da população da comunidade europeia, a variação da percentagem de mulheres nos diferentes grupos etários é considerável: no grupo das mulheres com menos de 20 anos existem 95 mulheres por cada 100 homens, enquanto nas octogenárias existem cerca de 221 mulheres por cada 100 homens. Esta variação é explicada, pela diferença na esperança de vida, onde as mulheres, tem aumentado significativamente, estando próxima dos 80 anos, ou seja, 6 anos acima da esperança média de vida dos homens (OMS, 2019).

Atualmente, falar de saúde é falar da vida, porque "a saúde está no centro da vida e tudo o que diz respeito à vida diz respeito à saúde" (Hesbeen, 2000, p.23). Estando no centro da vida, a saúde faz parte da existência de cada um e, tal como não há vida, mas vidas, também não podemos dizer que existe (ou não) saúde, pois cada pessoa é quem perceciona a sua saúde de acordo com o seu projeto, as suas crenças, ou seus ideais. Segundo Honoré (1996), assumimos a saúde "como nossa" enquanto existimos, pois ela é condição de existência. Contudo, se até há alguns anos a saúde era a mera ausência da doença, e se a longevidade era, em si, um bem, hoje a longevidade já não é um fator essencial a uma "boa saúde". 


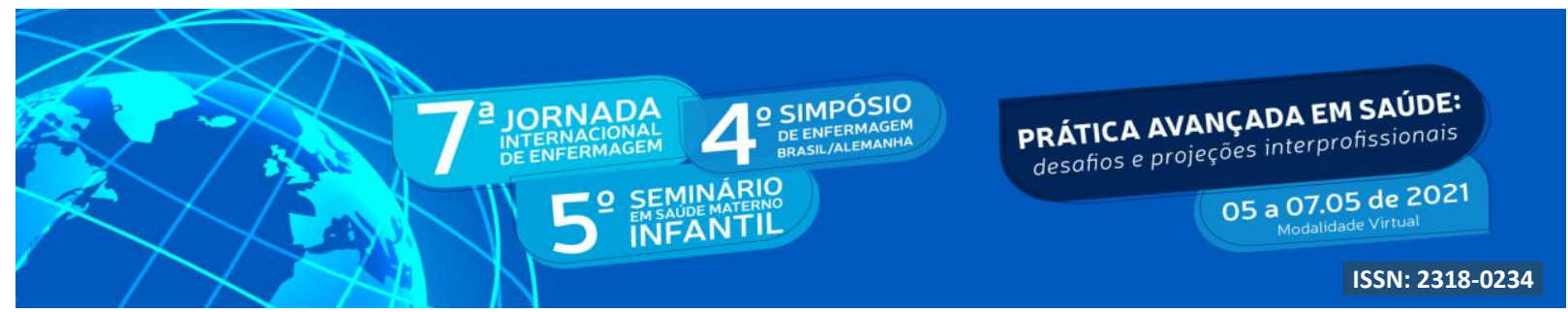

A conceção de saúde não depende apenas do facto de saber o que ela é, ou construir teorias em seu redor, mas essencialmente de verificar como se manifesta de uma forma renovada e que em cada indivíduo assume um aspeto particular. A saúde não passa só pelo saber (nível cognitivo) e sentir (nível emocional), mas também pela compreensão de sentido, o que nos permite atribuir-lhe um determinado valor e significado (nível cultural). Esta atribuição semântica evolui com a experiência, é determinada pelo tempo. A saúde é também uma noção relacional, já que a saúde de uma pessoa pode ser objeto de experiência de outra. A experiência de saúde é uma noção fluída, subjetiva, difícil de explicar; muda com o tempo, os contextos, as relações, tornando-se uma noção completa (Honoré, 2002).

As Estratégias para a Saúde procuram salvaguardar incondicionalmente a dignidade humana, a solidariedade e a justiça social na realização da saúde, os princípios éticos na prática clínica e nas decisões individuais e coletivas sobre a saúde.

Tendo presente que persistem modelos de socialização diferentes em função do sexo, urge refletir até que ponto as experiências de saúde e de doença podem apresentar significados diferentes em função do género, sendo necessário salvaguardar que aquelas experiências de saúde e de doença também variam entre as diversas configurações culturais.

\section{OBJETIVO}

O objetivo deste estudo passou por aproximar-nos da realidade do estado de saúde das mulheres cabo-verdianas, partindo da visão que têm de si próprias, pelo que procurámos conhecer as caracteristicas sociodemográficas e de saúde das mulheres cabo-verdianas.

\section{METODOLOGIA}

Foi realizado um estudo de natureza quantitativa, descritivo e transversal, a 104 mulheres cabo-verdianas, com recurso a uma amostragem não probabilistica de conveniência. $\mathrm{O}$ acesso a estas mulheres fez-se através da técnica de amostragem da bola de neve (snowball sampling). A amostra por bola de neve é uma técnica de amostragem não probabilística onde os indivíduos selecionados, para serem estudados, convidam novos participantes da sua rede 


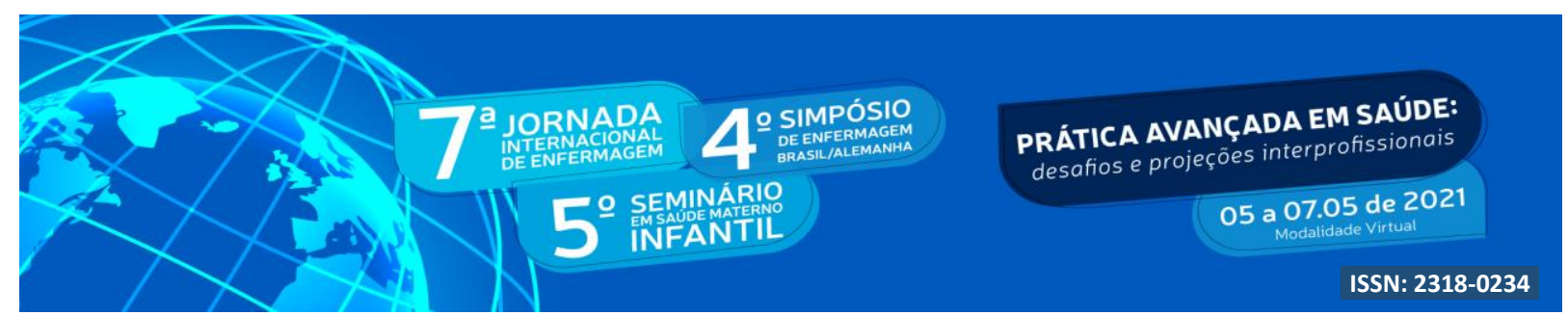

de amigos e conhecidos.

O estudo realizou-se através da aplicação, num momento único, de um questionário constituído por dados sociodemográficos (variáveis idade, estado civil, escolaridade e situação laboral) e dados relativos à perceção da saúde por parte das mulheres que participaram no mesmo. Para esse efeito foram utilizados o Questionário de Perceção Acerca da Saúde (John Ware, 1976) e a Escala de Bem-Estar Psicológico Geral (Dupuy, 1984). A recolha de dados decorreu entre os meses de dezembro de 2019 a julho de 2020.

A análise dos dados foi feita com recurso à versão 27 do Statistical Package for the Social Sciences (SPSS Inc. Chicago, Illinois), foi utilizada a estatística descritiva, mais concretamente as frequências absolutas $\left(\mathrm{n}^{\circ}\right)$, as frequências relativas $(\%)$, médias $(\bar{X})$, modas (Mo), medianas (Md), Desvio Padrão ( $\sigma$ ), Valor Mínimo (Xmin.) e Valor Máximo (Xmáx.), tendo em conta os dados em análise. Foram tidos em consideração os procedimentos formais e éticos, nomeadamente o pedido de parecer à Comissão de Ética da Unidade de Investigação em Ciências da Saúde: Enfermagem da Escola Superior de Enfermagem de Coimbra, que emitiu parecer positivo (Parecer $\left.\mathrm{n}^{\circ} \mathrm{P} 573\right)$ ). O questionário foi elaborado com recurso aos meios digitais, onde primeiramente constava a informação relativa à investigação e a secção relativa ao consentimento informado. Não era possível avançar no questionário sem o preenchimento prévio e aceitação do consentimento informado. O questionário foi partilhado online através de plataformas digitais, pelo que a participação das mulheres foi totalmente livre.

\section{RESULTADOS E DISCUSSÃO}

No que concerne aos dados sociodemográficos verifica-se que a média de idades das nossas participantes é de 36 anos, 54,8\% (57) são solteiras e 39,4\% (41) são casadas, somente $4,8 \%$ (5) referem que o seu estado civil é separadas/divorciadas (Gráfico 1). É muito interessante de verificar, no que toca à escolaridade, que mais de metade das mulheres caboverdianas $(55,8 \% ; 58)$ que participaram neste estudo possuem uma licenciatura, sendo que 12,5\% (13) possuem o ensino secundário completo e também 12,5\% (13) o nono ano de escolaridade. Somente 1\% (1) das mulheres possuem um mestrado (Gráfico 2). 


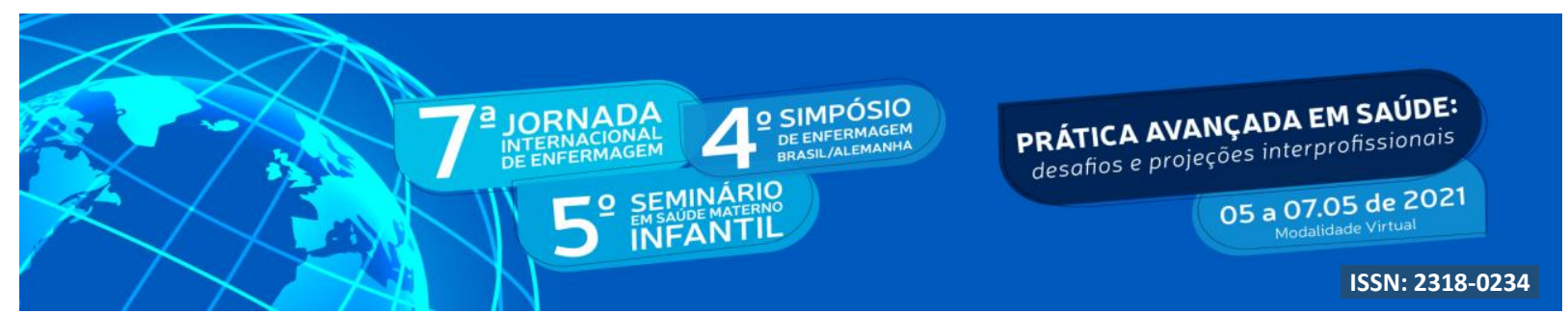

Gráfico 1: Dados Sociodemográficos - Estado Civil

\section{Estado Civil}

80

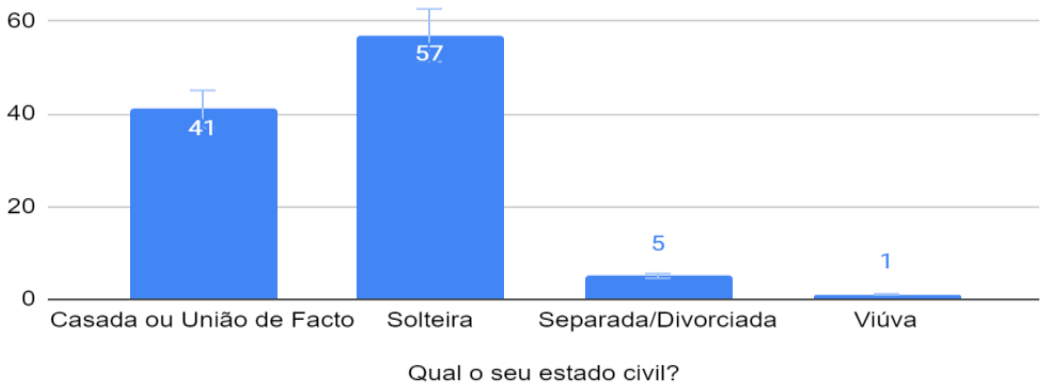

Gráfico 2: Dados Sociodemográficos - Escolaridade

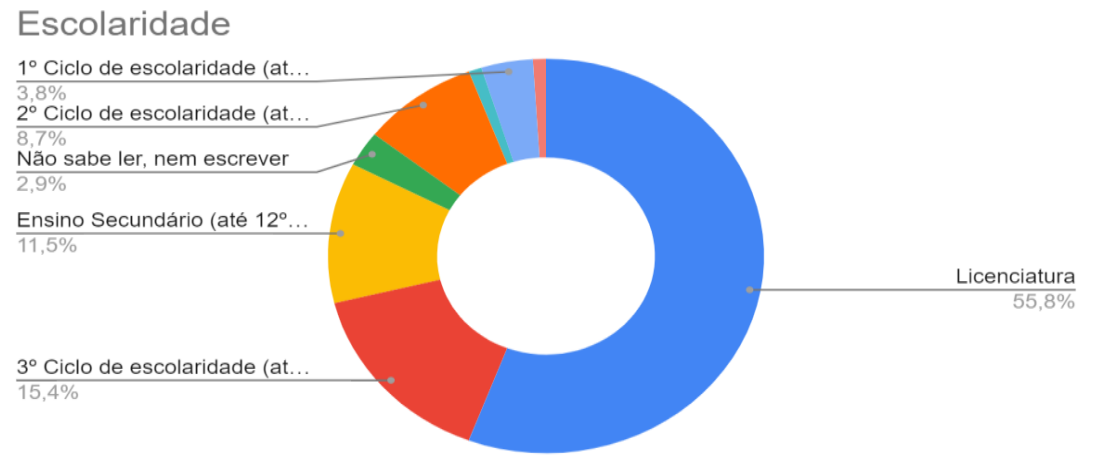

No que concerne à perceção que estas mulheres têm sobre o seu estado de saúde verifica-se que 53,8\% (56) consideram que de acordo com os médicos por si consultados, a sua saúde está agora excelente, embora 10,6\% (11) consideram que não. As mulheres caboverdianas referem em 13,5\% (14) que a têm a percecão de adoecer mais facilmente do que as outras pessoas, ao contrário de $51 \%$ (53), sendo que 19,2\% (20) entendem que a probabilidade em ficar doente, no futuro, é elevado. Das mulheres participantes 20,2\% (21) indicam que não gostam de ir ao médico e $28,8 \%$ (30) referem preocupação com a sua saúde atual, no momento que participaram no estudo. As mulheres cabo-verdianas afirmam em 37,5\% (39), que não são atualmente tão saudáveis, como no passado e 51,9\% (54) referem que quando estão doentes, tentam continuar com a sua vida quotidiana de costume, 


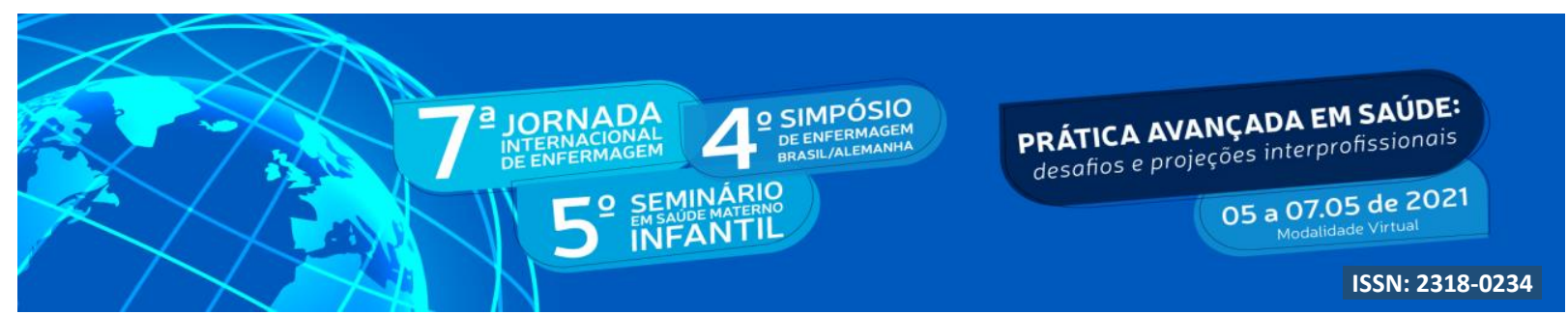

verificando-se que 54,8\% (57) destas mulheres perceciona que o seu corpo parece resistir muito bem à doença. Os dados que obtivemos mostram-nos que 33,7\% (35) destas mulheres quando estão doentes tentam guardar esse facto somente para elas e 65,4\% (68) sentem que vão adoecer (Gráfico 3) em que, 33,7\% (35) enfatizam que ultimamente se têm sentido adoentadas. A maioria das mulheres $(72,1 \%, 75)$ enunciam que quando se sentem a adoecer lutam contra essa sensação geral de mal-estar (Gráfico 4).

Gráfico 3: Perceção de Saúde

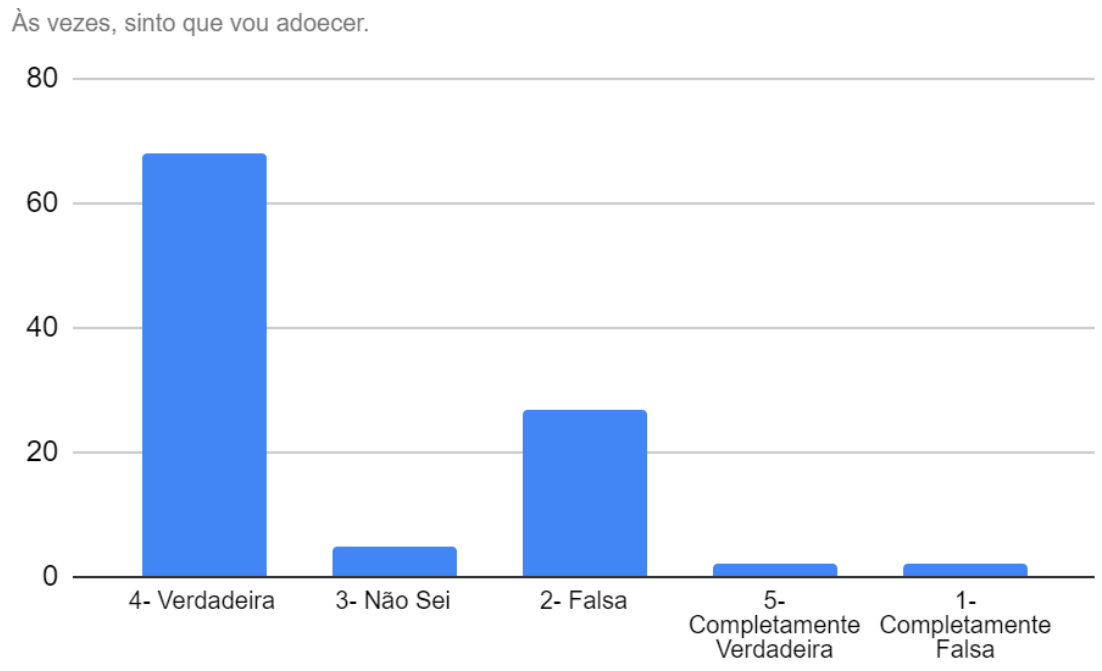

Gráfico 4: Perceção de Saúde

Quando me sinto a adoecer, luto contra isso.

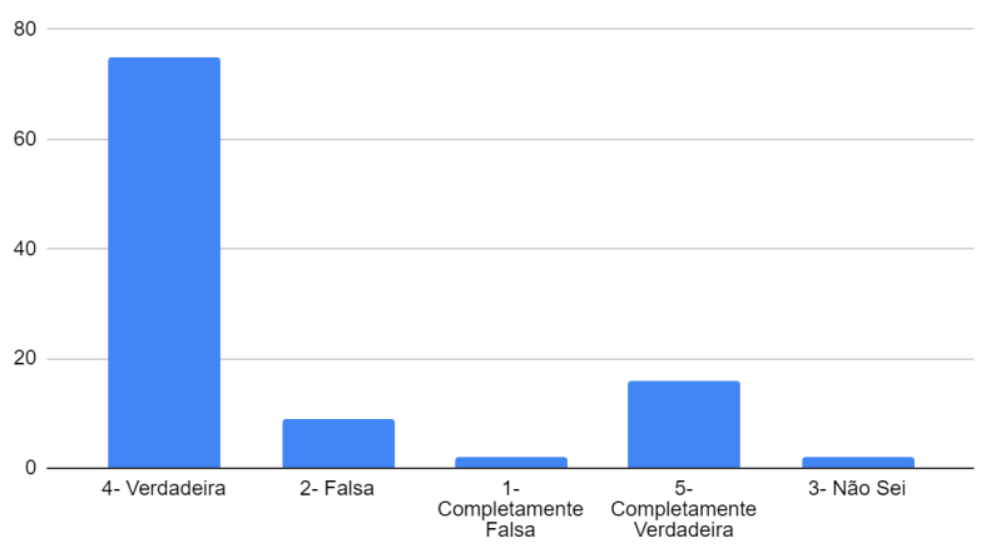




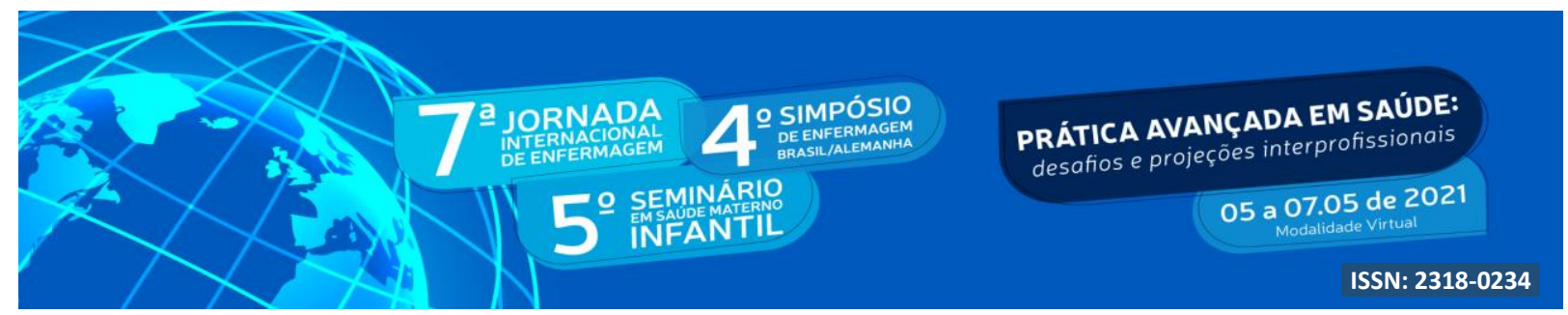

Em 2000, os Estados-Membros das Nações Unidas comprometeram-se a alcançar oito objetivos principais de desenvolvimento até 2015 conhecido como os Objetivos de Desenvolvimento do Milênio (ODM), no que concerne às questões da igualdade de género (ONU Mulheres, 2019). Embora tenha havido avanços em todos eles, globalmente não se atingiram os objetivos definidos. Apesar dos ganhos ao nivel da morbi e mortalidade materna e ao nivel da escolaridade, verifica-se que por exemplo, a mortalidade materna diminuiu $45 \%$, no entanto, a meta estimada era $75 \%$. Não obstante dos avanços na cobertura do tratamento de HIV / AIDS, malária e outras doenças, o HIV infeta 50 mulheres a cada hora (ONU Mulheres, 2019).

No ano de 2009, no relatório da OMS 'Mulheres e Saúde - Dados de Hoje, Agenda de Amanhã' verificou-se a existência de amplas e persistentes desigualdades entre os homens e mulheres, onde a questão das doenças crónicas, doenças infetocontagiosas e doenças associadas ao sistema reprodutor, lesões e transtornos mentais, fazem parte do quotidiano de muitas mulheres no mundo, vivenciadas em sofrimento e em silêncio. Em paises de baixos rendimentos a doença é experienciada pelas mulheres com aceitação, sofrimento e resignação (OMS, 2009), o que vai ao encontro dos dados por nós encontrados, onde as mulheres caboverdianas referem que quando estão doentes, tentam continuar com a sua vida quotidiana, em que o seu corpo parece resistir muito bem à doença e quando estão doentes tentam guardar esse facto somente para elas, procurando lutar contra isso. Verifica-se ainda que algumas das mulheres que participaram no estudo percecionam e sentem que estão doentes, referindo que ultimamente se têm sentido mal.

\section{CONCLUSÃO}

O estudo mostra que é necessário investir na saúde das mulheres, principalmente em países onde as desigualdades entre homens e mulheres são ainda significativas. Necessitamos igualmente, de criar condições equitativas de nascimento para meninas e meninos, 


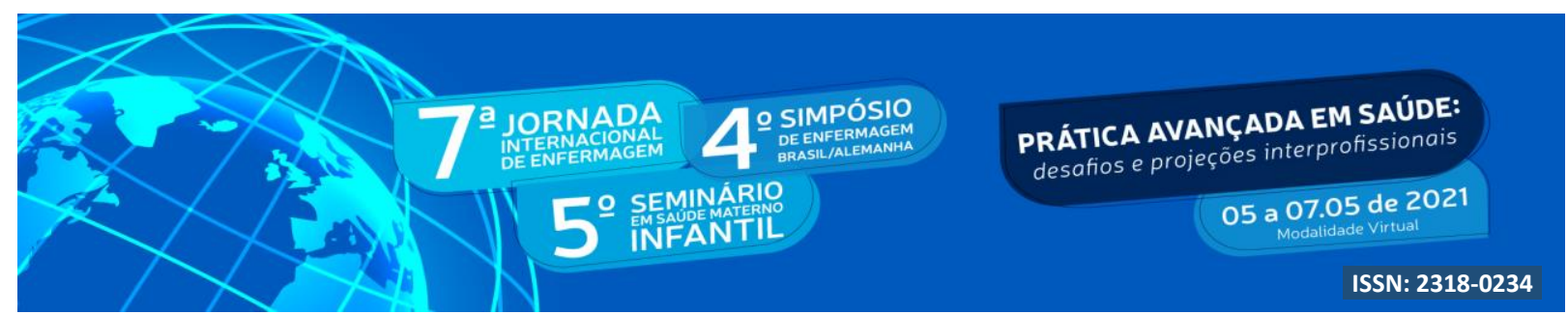

promovendo uma vivência salutar da adolescência, idade adulta e velhice. Os enfermeiros estão nas melhores condições não só para promover cuidados ao longo do ciclo vital das mulheres como trabalhar na comunidade junto das populações as questões da desigualdade de género, morbilidade e mortalidade na mulher, violência contra as mulheres, sexualidade protegida e saudável, entre outras temáticas. É nossa convicção que, a elaboração e implementação de uma agenda de saúde para a mulher em Cabo Verde e em outros países, permitirá superar desigualdades e melhorar a saúde de todos.

\section{REFERÊNCIAS}

DUPUY, H. J. The Psychological General Well-Being (PGWB) Index. In N.K. Wenger, M.E. Mattson, C.D. Furberg, \& J. Elinson (Eds.), Assessment of quality of life in clinical trials of cardiovascular therapies (pp. 170-183). New York: LeJacq, 1984.

HESBEEN, W. Cuidar no hospital: enquadrar os cuidados de enfermagem numa perspectiva de cuidar. Loures: Lusociência, 2000.

HONORÉ, B. A saúde em projecto. Loures: Lusociência, 2002.

ONU MULHERES. EI Progreso de las Mujeres en el Mundo 2019-2020. ONU Mujeres, 2019.

OMS. Mulheres e Saúde - Dados de Hoje, Agenda de Amanhã. Organização Mundial da Saúde, 2009.

WARE, J. E. The assessment of health status. In Aiken LH, Mechanic D, editors. Applications of social sciences to clinical medicine and health policy. New Brunswick, NJ: Rutgers University Press, 1976. 